\title{
BMC Ear, Nose and Throat Disorders reviewer acknowledgement 2014
}

Magdalena Morawska

\section{Contributing reviewers}

The editors of BMC Ear, Nose and Throat Disorders would like to thank all of our reviewers who have contributed to the journal in Volume 14 (2014).

Ilona Anderson
Austria
Minakshi Bhardwaj
India
Marc Braem
Belgium
Itzhak Brook
USA
José Cameselle-Teijeiro
Spain
Peter W. Dettmar
UK
Y.P. Peter Di
USA
Wouter Dreschler
Netherlands
Caterina Finizia
Sweden
Elizabeth Fitzpatrick
Canada
Noriyuki Fujima
Japan
Karyn Galvin
Australia

$\begin{array}{ll}\text { Miguel Goncalves } & \text { David J Lim } \\ \text { UK } & \text { USA } \\ \text { Hasantha Gunasekera } & \text { Fernanda Mariano } \\ \text { Australia } & \text { Brazil } \\ \text { Hervé Haas } & \text { Elismauro Mendonca } \\ \text { France } & \text { Brazil } \\ \text { Stefan Hegemann } & \text { Ranko Mladina } \\ \text { Switzerland } & \text { Croatia } \\ \text { Sebastian Hoth } & \text { Markus Moessner } \\ \text { Germany } & \text { Germany } \\ \text { Hiroaki Ichijo } & \text { Tsutomu Nakashima } \\ \text { Japan } & \text { Japan } \\ \text { Takao Imai } & \text { George Noussios } \\ \text { Japan } & \text { Greece } \\ \text { Fikret Kasapoglu } & \text { Toyoaki Ohbuchi } \\ \text { Turkey } & \text { Japan } \\ \text { Tejs Klug } & \text { Bolajoko O. Olusanya } \\ \text { Denmark } & \text { Nigeria } \\ \text { Masahiro Komori } & \text { Fredrik Petersson } \\ \text { Japan } & \text { Singapore } \\ \text { Kenji Kondo } & \text { Antonio Schindler } \\ \text { Japan } & \text { Italy } \\ \text { Seung-Han Lee } & \text { Aiden Shearer } \\ \text { South Korea } & \text { USA }\end{array}$

Correspondence: Magdalena.Morawska@biomedcentral.com

BioMed Central, Floor 6, 236 Gray's Inn Road, London WC1X 8HB, UK 
Sivakumaran Theru Arumugam USA

Valentino Valentini

Italy

Fabiana Valera

Brazil
Katrien Vermeire

Belgium

Petros Vlastarakos

UK

Che-Ming Wu

Taiwan
Hasan Yasan

Turkey

Bülent Yazici

Turkey 